\section{BMJ Open} Ophthalmology

\title{
Genetic biomarkers in the VEGF pathway predicting response to anti- VEGF therapy in age-related macular degeneration
}

Irina Balikova (D) ,,2 Laurence Postelmans, ${ }^{2}$ Brigitte Pasteels, ${ }^{2}$ Pascale Coquelet, ${ }^{2}$ Janet Catherine, ${ }^{2}$ Azra Efendic, ${ }^{2}$ Yoshikatsu Hosoda, ${ }^{3}$ Masahiro Miyake, ${ }^{3}$ Kenji Yamashiro, ${ }^{3,4}$ ANGEL study group members, Bernard Thienpont,, Diether Lambrechts ${ }^{5,7}$

\section{ABSTRACT}

Objective Age-related macular degeneration (ARMD) is a leading cause of visual impairment. Intravitreal injections of anti-vascular endothelial growth factor (VEGF) are the standard treatment for wet ARMD. There is however, variability in patient responses, suggesting patient-specific factors influencing drug efficacy. We tested whether single nucleotide polymorphisms (SNPs) in genes encoding VEGF pathway members contribute to therapy response.

Methods and analysis A retrospective cohort of 281 European wet ARMD patients treated with anti-VEGF was genotyped for 138 tagging SNPs in the VEGF pathway. Per patient, we collected best corrected visual acuity at baseline, after three loading injections and at 12 months. We also registered the injection number and changes in retinal morphology after three loading injections (central foveal thickness (CFT), intraretinal cysts and serous neuroepithelium detachment). Changes in CFT after 3 months were our primary outcome measure. Association of SNPs to response was assessed by binomial logistic regression. Replication was attempted by associating visual acuity changes to genotypes in an independent Japanese cohort.

Results Association with treatment response was detected for seven SNPs, including in FLT4 (rs55667289: $\mathrm{OR}=0.746,95 \% \mathrm{Cl} 0.63$ to $0.88, \mathrm{p}=0.0005$ ) and $\mathrm{KDR}$ (rs7691507: $0 \mathrm{R}=1.056,95 \% \mathrm{Cl} 1.02$ to $1.10, \mathrm{p}=0.005$; and rs2305945: $0 \mathrm{R}=0.963,95 \% \mathrm{Cl} 0.93$ to 1.00 , $\mathrm{p}=0.0472$ ). Only association with rs55667289 in FLT4 survived multiple testing correction. This SNP was unavailable for testing in the replication cohort. Of six SNPS tested for replication, one was significant although not after multiple testing correction.

Conclusion Identifying genetic variants that define treatment response can help to develop individualised therapeutic approaches for wet ARMD patients and may point towards new targets in non-responders. permitted under CC BY-NC. No commercial re-use. See rights and permissions. Published by BMJ.

For numbered affiliations see end of article.

Correspondence to Dr Irina Balikova; Irina. Balikova@uzleuven.be

\section{Key messages}

What is already known about this subject ?

- Genetic factors influence the response to antivascular endothelial growth factor treatment in patients with age-related macular degeneration (ARMD).

\section{What are the new findings?}

We found association between single nucleotide polymorphisms in FLT4, KDR and VEGFC and treatment response.

How might these results change the focus of clinical practice in the future?

- These results can help in developing new treatment targets for ARMD.

neovascular form, which involves the development of new blood vessels that are poorly formed, leaky and structurally abnormal (choroidal neovascularisation, CNV). CNV can lead to retinal haemorrhage and accumulation of fluid within and under the retina and the retinal pigment epithelium. ${ }^{2}$

Neovascularisation in ARMD is shown to be elicited by different growth factors including placental, platelet-derived, fibroblast (FGF) and transforming growth factors, tumour necrosis factor, eotaxin and most prominently, by the vascular endothelial growth factor (VEGF). ${ }^{3}$ A long-standing therapeutic aim is therefore to block VEGF signalling. This can be achieved by injections of VEGF-scavenging molecules, that prevent VEGF from binding and activating its receptor. Indeed, injections with anti-VEGF monoclonal antibodies such as ranibizumab (Lucentis) - a FAB fragment, bevacizumab (Avastin) - a full antibody or with aflibercept (Eylea) - anti-VEGF recombinant protein, are the standard treatment for wet ARMD ${ }^{4-6}$ While the majority of patients 
respond well to this treatment and regain visual acuity, about $10 \%-20 \%$ of patients fail to respond for reasons that are poorly understood. ${ }^{47}$

A number of studies have investigated the influence of genetic factors on the response to anti-VEGF treatment in neovascular ARMD, as recently reviewed by Lorés-Motta and colleagues. ${ }^{8}$ These include polymorphisms in genes previously shown to play role in the pathogenesis of ARMD, such as CFH, ARMS, HTRA1, C3, CFB, ARMS2 and SERPINF1, which have been extensively studied. ${ }^{9}{ }^{10}$ For example, a polymorphism in $\mathrm{CFH}$ (rs1061170/Y402H) has been associated with improved outcome, ${ }^{11}$ although this was not found in the IVAN and CATT studies (studies comparing the effectiveness of Ranibizumab versus Bevacizumab for treating neovascular AMD).$^{12}{ }^{13}$ Other groups have investigated polymorphisms in genes encoding components of the VEGF pathway, showing for example association of single nucleotide polymorphisms (SNPs) in VEGFR2 (rs4576072 and rs6828477) with better visual outcome while others did not find correlation. ${ }^{13-15}$ Moreover, in a recent metaanalysis, anti-VEGF treatment was found to be more effective in patients homozygous for the VEGFA rs833061 minor allele $\mathrm{C}(\mathrm{OR}=2.362) .{ }^{16}$ In addition to these more targeted approaches, genome-wide association studies allow for an unselected investigation of genetic factors related to the response to anti VEGF treatment. Riaz and colleagues used pooled DNAs and reported a correlation between worsened response and rs4910623, a SNP in the promoter region of OR52B4. ${ }^{17} \mathrm{~A}$ recent report including 919 Japanese patients showed possible association of four SNPs (rs17822656, rs76150532, rs17296444 and rs75165563) with lack of response. ${ }^{18}$ The heterogeneity of the outcome of these studies indicates a need of further studies to understand the effect of genotype on the anti-VEGF treatment. ${ }^{8}$

Anti-VEGF treatment is also used in the cancer clinic, where non-response has similarly been observed. ${ }^{19}$ Recent studies have identified germline genetic variants that reduce VEGF pathway activity, leading to the appropriation or initiation of alternative neo-angiogenic pathways such as those driven by FGF or placental growth factor (PGF). Since alternative pathways are more active in these patients, they fail to show an appropriate response to anti-VEGF, an explanation supported by work in mice. ${ }^{20}$ Based on these findings in cancer we hypothesised that similar mechanism might apply to the non-response to anti-VEGF therapy of ARMD patients. Therefore, we tested whether single nucleotide variants in genes encoding the VEGF pathway members can predict the therapeutic response to anti-VEGF treatment in ARMD.

\section{METHODS}

\section{Patient population}

The study was conducted following the tenet of Helsinki and was approved by the local ethic committee. After informed consent was obtained, consecutive patients followed between 2006 and 2014 at a single reference ARMD centre (Ophthalmology Department of the Brugmann University Hospital, Brussels) were retrospectively included in this study. Diagnosis of new subfoveal neovascular ARMD was for all patients made by established specialists and was based on multimodal imaging: Optical Coherence Tomography (OCT), fluorescein and indocyanine green angiographies (FA and ICG). All patients had received three loading anti-VEGF injections of Lucentis and then pro re nata (PRN) treatment with Lucentis or Avastin for at least 1 year as described. ${ }^{21}{ }^{22}$ Specifically, if non-response to Lucentis was observed after the fourth injection, the product was switched to Avastin. Patients who received ARMD treatment other than anti-VEGF, or who were on treatment for less than 1 year were excluded. Note that Eylea as an ARMD drug became available later. To avoid unwanted variation, the few patients treated with Eylea were excluded from the study. The treatment was monitored with control visits initially after the three loading injections, and then at monthly intervals. During these visits, best corrected visual acuity (BCVA), OCT, dilated colour pictures and examination of the eye fundus were performed. If reactivation was suspected by the clinical data or/and imaging, FA and if considered necessary ICG was performed. For patients having developed bilateral neovascular ARMD, only the response of the first treated eye was included in our analysis.

As a validation analysis, we assessed a previously genotyped cohort of 252 ARMD Japanese patients, similarly treated with monthly Lucentis injections. ${ }^{23}$ We assessed association of SNPs with changes in visual acuity, again at 3 months, as also these patients were received PRN treatment after 3 months. The association with visual acuity as an outcome measure is suboptimal, but was chosen because central foveal thickness (CFT) measurements were unavailable.

\section{Patient and public involvement}

This study aimed to improve our assessment of whether or not patients with ARMD will respond to anti-VEGF therapy. All patients were informed of the study and opted to be included. Patients are aware that they will not be recontacted to be informed about the study outcome.

\section{Phenotyping}

For each patient, the following variables were recorded: gender, age at treatment onset, number of anti-VEGF injections and the type of product received during the 12 first months of treatment, BCVA and CFT $(\mu \mathrm{m})$ at treatment onset and after 3 and 12 months; and morphological activity signs of neovascular ARMD, namely intraretinal cysts (IC) and subretinal fluid (SRF). Morphological ARMD signs were scored as present or absent at treatment onset, and re-evaluated after the three loading injections, scoring complete resolution as 0 , decreased amounts without full resolution as -1 , and unchanged or increased amounts as -2 . CFT was measured on Spectralis OCT (Heidelberg Engineering) or on Stratus OCT 


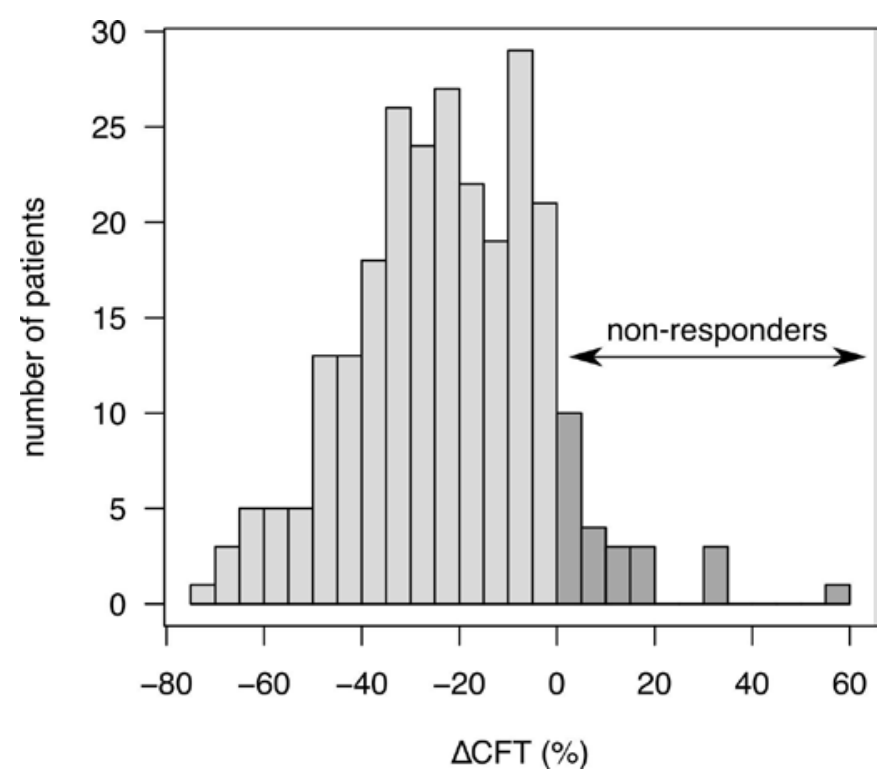

Figure 1 Distribution of the patients according to the difference in central foveal thickness $(\triangle C F T)$ after 3 months. Patients showing no change or an increase in the CFT were defined as non-responders.

(Zeiss). The retinal layer segmentation was controlled for each patient and readjusted when necessary. CFT measurements from both devices were rendered comparable by transformation, as described previously. ${ }^{24}$

\section{Outcome}

Our primary outcome measure was the change in CFT at 3 months after treatment onset. Nominal CFT changes are highly dependent on baseline CFT values, with high CFT before treatment onset being often associated with larger decreases following treatment. Continuous CFT changes hence do not capture treatment response well, and we therefore categorised the CFT data. We considered patients showing an increased CFT (CFT at 3 months being higher but not equal to the CFT at start), as non-responding (figure 1), in line with other studies. ${ }^{25}$ We note that such categorisation may cause loss of statistical information, as stratifying continuous data into categories can reduce power. In an independent analysis, we used morphological signs (changes in IC and SRF) as an additional response measure. Secondary outcomes were the change in CFT and the number of injections at 12 months, as well as the change in visual acuity at 3 and 12 months.

\section{Genotyping}

DNA was extracted from the peripheral blood of the patients using the QIAGEN Blood and Tissue kit according to manufacturer's instructions. Sequenom Maldi-tof analysis was used to genotype this DNA for common tagging SNPs in genes encoding VEGF pathway components as described. A target SNP was considered as genotyped successfully if over $98 \%$ of samples yielded a signal. Samples quality was considered sufficient if over $90 \%$ of SNPs were genotyped.

\section{Analysis}

SNPs were coded according to a dominant model, with homozygosity of the major allele, heterozygosity and homozygosity of minor allele being encoded, respectively as 0,1 and 1. Also a codominant model was considered, coding the same genetic constellations as 0,1 and 2. All calculations were done using $\mathrm{R}$ (V.3.3.1). Association of SNPs to therapy response was assessed by binomial logistic regression. A prespecified threshold of $\mathrm{p}$ values below 0.05 before multiple testing correction were considered to be significant.

\section{RESULTS}

\section{Patients and phenotyping}

301 patients were sampled. Of these, 20 patients were excluded: seven had very incomplete files (over half of the phenotypes missing), three had incomplete follow-up, two were treated in part elsewhere, two did not complete their treatment protocol, one received laser treatment prior to intravitreal anti-VEGF injections, one received injection of triamcinolone (Kenacort) and four failed genotyping. Hence, 281 patients were analysed. Phenotypes of these 281 patients are summarised in table 1. Importantly, 255 had CFT measurements at treatment onset as well as after 3 months, and 244 were also measured for CFT after 12 months. The vast majority of these (231 of 255; $91 \%$ and 200 of 244; 82\%) showed treatment response after 3 and 12 months. Responders more frequently also showed improved morphological ARMD signs (figure 2A,B). In contrast, although the change in BCVA was higher in responders than in non-responders, this failed to reach significance $(p=0.07)$, in line with earlier reports suggesting that changes in BCVA poorly reflect therapeutic response. ${ }^{2627}$ Age and gender distributions between responders and non-responders were similar. Finally, response appeared mostly durable, as CFT after 12 months remained significantly improved in responders but did not improve significantly across non-responders (figure 2C). Nevertheless, some patients non-responsive after 3 months did show response after 12 months $(n=11)$. Others on the other hand failed to show durable responses, showing an initially decreased CFT that was, after 12 months, increased versus the treatment onset $(n=29)$.

\section{Genotyping}

Overall, 177 SNPs were analysed. Of these, genotyping failed for three (rs1075952, rs1485766 and rs1678607), and two SNPs were not informative (all homozygous; rs35832528 and rs11549467). Using stringent criteria, we excluded additional SNPs for not being in HardyWeinberg equilibrium (rs11689649, rs13359473, rs2230054, rs2241906, rs2491417, rs3741403, rs4953344, rs6025, rs6418686, rs6527518, rs6632474, rs6753127, rs7565341 and rs7594278). Hence, a total of 158 informative SNPs were included in our analyses. Technical genotyping replicates were run for five patients and showed $100 \%$ concordance. 
Table 1 Patient characteristics. Shown are mean \pm SD, and the data range between parentheses

\begin{tabular}{|c|c|c|c|c|}
\hline Age & $77 \pm 8.9$ years $(58-97)$ & \multirow{2}{*}{\multicolumn{2}{|c|}{$36 \%$ men }} & \\
\hline Gender & $64 \%$ women & & & \\
\hline \multicolumn{5}{|l|}{ Number of injections after 1 year } \\
\hline All combined & \multicolumn{4}{|l|}{$6.0 \pm 2.3(3-11)$} \\
\hline Lucentis & \multicolumn{4}{|l|}{$5.5 \pm 1.9(3-9)$} \\
\hline \multirow[t]{2}{*}{ Avastin } & \multicolumn{4}{|l|}{$0.5 \pm 1.3(0-7)$} \\
\hline & Onset & \multicolumn{2}{|l|}{3 months } & 1 year \\
\hline Visual acuity (Snellen) & $0.44 \pm 0.25$ & \multicolumn{2}{|l|}{$0.56 \pm 0.28$} & $0.55 \pm 0.31$ \\
\hline Central foveal thickness & $421 \pm 136(145-933)$ & \multicolumn{2}{|c|}{$313 \pm 98(145-906)$} & $335 \pm 110(155-841)$ \\
\hline \multirow[t]{2}{*}{ Morphological signs } & & \multicolumn{3}{|c|}{ After 3 months } \\
\hline & Presence at onset (\%) & Resolved (\%) & Decreased (\%) & $\begin{array}{l}\text { Stable/worsened } \\
\text { (\%) }\end{array}$ \\
\hline Intraretinal cysts & 99 & 51 & 38 & 11 \\
\hline Subretinal fluid & 83 & 62 & 31 & 12 \\
\hline Pigment epithelial detachment & 94 & 24 & 66 & 10 \\
\hline
\end{tabular}

\section{Association analysis}

The patients were classified as responders or nonresponders based on the change of CFT after the three loading injections.

Association of genotypes with treatment response was measured using a generalised linear model, correcting for the patient's age and gender. Overall, seven SNPs showed a significant association with treatment outcome after 3 months, and 10 SNPs with outcome after 12 months $(\mathrm{p}<0.05$; table 2; figure $3 \mathrm{~A})$. Of these, two were also significantly associated with the occurrence of structural changes after 3 months (rs55667289 and rs9513070), and only one with improved visual acuity (figure 3B). The total number of injections was associated with three of these SNPs (online supplementary table 2). We also tested correlations using an additive (codominant) model, and recovered 15 SNPs, 10 of which match those significant in the dominant model. This indicates that
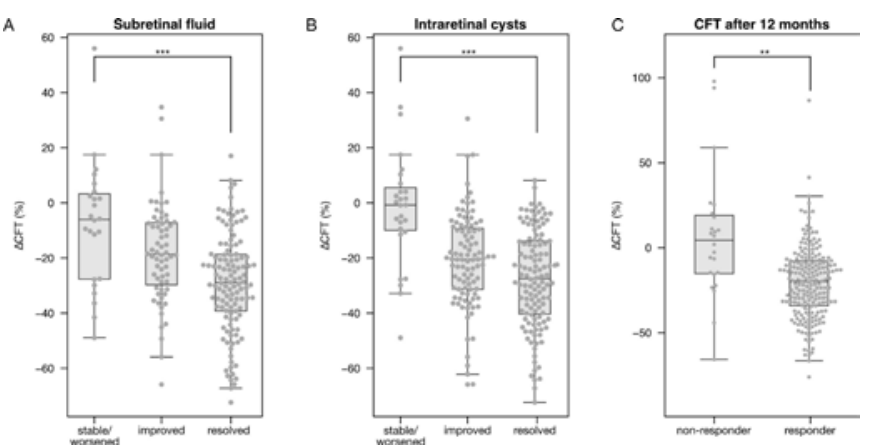

Figure 2 Boxplots showing the difference in central foveal thickness $(\triangle \mathrm{CFT})$, after 3 months in patients with subretinal fluid (A) or intraretinal cysts (B) at the study onset, that after 3 months either did not improve, improved or was resolved, and after 12 months in patients that did or did not show a decrease in CFT after 3 months (respectively, responder and non-responder). ${ }^{* \star *} p<0.001 ;{ }^{* *} p<0.01$. a dominant or codominant model yield only moderate differences.

\section{Analysis of an independent cohort}

To further assess these findings, we reanalysed data from an independent cohort of 252 patients of Japanese descent that was previously described. ${ }^{23}$ In this study the outcome was defined by change in the visual acuity after 3 months on treatment. The six most significant SNPs from our analysis $(\mathrm{p}<0.01)$ were selected. The associated genotypes of five of these could be measured or imputed in a genome-wide assay, and only for rs55667289 no data were available. ${ }^{23}$ These genotypes were next correlated in a linear model with treatment response, measured using change in BCVA, while correcting for age and gender as above. Interestingly, while association for only one of these five SNPs attained significance (rs2046462; $p=0.019$ ), the direction of the association was the same for all five SNPs, thus providing moderate support for the validity of our findings (table 3). Nevertheless, none of the $p$ values survived multiple testing correction.

\section{DISCUSSION}

In our study, we assessed associations in patients with neovascular ARMD between the response to antiVEGF treatment and SNPs in components of the VEGF pathway. We genotyped tagging SNPs near a range of genes encoding hypoxia sensing proteins (PHD1, PHD2, PHD3, FIH and VHL) and transcription factors (HIF1A, HIF2A), as well as angiogenic growth factors (VEGFA, VEGFB, VEGFC, VEGFD, IL8, FGF and PGF) and receptors (VEGFR1, VEGFR2 and VEGFR3). To discriminate responders from non-responders, we measured the change in the CFT, as well as a combination of morphological features. The use of CFT and other morphological signs allows for robust definition of response as it is directly proximal to the underlying pathology, that is, 
Table 2 SNPs significantly correlating with CFT response 3 or 12 months after treatment onset (primary and secondary outcome measure) in a dominant model, and $p$ values of correlation with response. Not significant $(p>0.05)$. The ORs for association are also tabulated, and represent the risk for non-response if the SNP is present at least once

\begin{tabular}{|c|c|c|c|c|c|c|}
\hline SNP & Gene & Genome position & Minor allele & $\begin{array}{l}\text { Minor allele frequency } \\
(\%)\end{array}$ & $P$ value & OR (95\% range) \\
\hline \multicolumn{7}{|c|}{ SNPS significantly correlating with CFT response after 3 months } \\
\hline rs55667289 & FLT4 & chr5:180621641 & $\mathrm{G}$ & 0.4 & 0.0005 & 0.746 (0.63 to 0.88$)$ \\
\hline rs7691507* & $K D R$ & chr4:55076834 & C & 20.7 & 0.0051 & 1.056 (1.02 to 1.10$)$ \\
\hline rs5758223 & MIR1281 & chr22:41093916 & G & 26.0 & 0.0115 & 0.954 (0.92 to 0.99$)$ \\
\hline rs9513070 & FLT1 & chr13:28305702 & G & 38.2 & 0.0204 & 1.045 (1.01 to 1.09$)$ \\
\hline rs4820431 & EP300 & chr22:41153917 & A & 35.8 & 0.0232 & 0.958 (0.92 to 0.99$)$ \\
\hline rs3025035 & VEGFA & chr6:43783622 & $\mathrm{T}$ & 6.3 & 0.0428 & 0.945 (0.90 to 1.00$)$ \\
\hline rs2305945 & $K D R$ & chr4:55105679 & $\mathrm{T}$ & 32.9 & 0.0472 & 0.963 (0.93 to 1.00$)$ \\
\hline \multicolumn{7}{|c|}{ SNPs significantly correlating with CFT response after 12 months } \\
\hline rs3775194 & VEGFC & chr4:176702723 & $\mathrm{G}$ & 39.2 & 0.0006 & 1.089 (1.04 to 1.14$)$ \\
\hline rs12054587 & VEGFC & chr4:176757527 & A & 9.7 & 0.0048 & 1.106 (1.03 to 1.19$)$ \\
\hline rs2046462 & VEGFC & chr4:176681324 & C & 29.6 & 0.0064 & 1.067 (1.02 to 1.12$)$ \\
\hline rs17086609 & FLT1 & chr13:28355574 & G & 33.3 & 0.0076 & 1.067 (1.02 to 1.12$)$ \\
\hline rs9551462 & FLT1 & chr13:28378967 & $\mathrm{T}$ & 35.0 & 0.0207 & 1.057 (1.01 to 1.11$)$ \\
\hline rs20551 & EP300 & chr22:41152004 & G & 28.0 & 0.0222 & 1.056 (1.01 to 1.11$)$ \\
\hline rs7691507* & $K D R$ & chr4:55076834 & C & 20.7 & 0.0256 & 1.058 (1.01 to 1.11$)$ \\
\hline rs2820037 & / & chr1:239273242 & $\mathrm{T}$ & 11.8 & 0.0267 & 0.934 (0.88 to 0.99$)$ \\
\hline rs10020464 & $K D R$ & chr4:55112903 & $\mathrm{T}$ & 32.0 & 0.0379 & 0.952 (0.91 to 1.00$)$ \\
\hline rs12510099 & VEGFC & chr4:176681802 & A & 10.5 & 0.0451 & 1.064 (1.00 to 1.13$)$ \\
\hline
\end{tabular}

CFT, central foveal thickness; SNP, single nucleotide polymorphism.

neovascularisation and the associated oedema. For this reason, they were preferred as an outcome measure over an improvement in vision, as the latter can fail to accurately reflect effects of the treatment if the initial vision is very good or very poor.

First, we observed an association between rs55667289 and lack of treatment response $\left(\mathrm{p}\right.$ value $\left.=5.4 \times 10^{-4}\right)$. This rare missense variant is located within FLT4 which encodes for the VEGF receptor 3, a tyrosine kinase receptor of
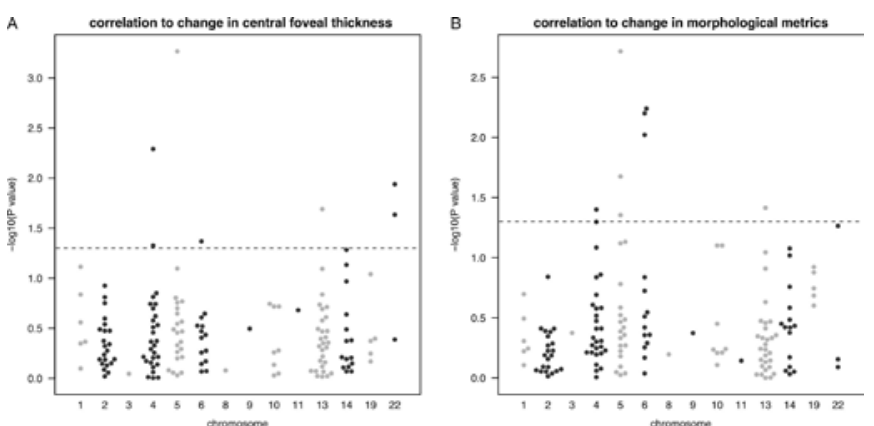

Figure 3 Manhattan plots showing $p$ values $(-\log 10-$ transformed; $Y$ axis) for the correlation between the frequencies of single nucleotide polymorphisms tested in this study $(n=156)$ and the change in $(A)$ central foveal thickness or in (B) morphological metrics (subretinal fluid and intraretinal cysts). $\mathrm{P}$ values are ranged by chromosome $(\mathrm{X}$ axis). The dotted line indicates $p=0.05$.
VEGFC and VEGFD. Lower VEGFR3 expression has previously been linked to a reduced response to sunitinib in clear cell renal cell carcinoma. ${ }^{28}$ VEGFR3-mediated signalling has mainly been studies in lymphangiogenesis, ${ }^{29}$ with mutations in this gene leading to Hereditary Lymphedema. ${ }^{30}$ VEGFR3 also regulates sprouting angiogenesis and the development of the cardiovascular network during the embryogenesis, and is expressed in vascular endothelial cells during pathological vessel remodelling. ${ }^{31}$ VEGFR3 expression has been detected in retinal blood vessels in mice, and was shown to restrict vascular permeability, with VEGFR3 loss being associated with an increased vascular leakage in the retina and other organs. Mechanistically, this increased permeability was ascribed to reduced adherent and tight junctions of vascular endothelial cells. ${ }^{32}$ Similar mechanisms could contribute to the poor anti-VEGF response in ARMD patients carrying the rs55667289 variant.

A second SNP associated with non-response is rs7691507. It is located near the $3^{\prime}$ end of the VEGFR2 gene, also known as KDR. This SNP was previously associated with the development of wet ARMD,${ }^{33}$ and also with sarcoidosis. ${ }^{34}$ No functional data are available for the effect of this SNP on VEGFR2 expression.

A third SNP of note was rs9513070, which is located intronically in VEGFR1. Interestingly, the A genotype that was associated here with a better response to anti-VEGF 
Table 3 Results of replication of five of the six SNPs showing linkage to response $(p<0.01)$ in the discovery cohort. Shown are results from the association between SNP genotype and change in BCVA (logMAR) after 3 months, as assessed using a linear model correcting for age and gender. Negative beta values indicate that the SNP is associated with a decreased vision. Also indicated is the direction of risk change in the discovery cohort, with increased and decreased corresponding to a higher and lower risk not to respond to anti-VEGF treatment, respectively. P values were not corrected for multiple testing

\begin{tabular}{llllll}
\hline SNP & Genotype & Risk in discovery cohort & Beta & SE & P value \\
\hline rs17086609 & Genotyped & Increased & -0.012 & 0.022 & 0.59 \\
rs2046462 & Imputed & Increased & -0.053 & 0.022 & 0.019 \\
rs7691507 & Genotyped & Increased & -0.052 & 0.040 & 0.19 \\
rs12054587 & Imputed & Decreased & 0.019 & 0.033 & 0.56 \\
rs3775194 & Imputed & Decreased & 0.019 & 0.027 & 0.49 \\
\hline
\end{tabular}

BCVA, best corrected visual acuity; SNP, single nucleotide polymorphism; VEGF, vascular endothelial growth factor.

treatment, has previously been associated to a better antiangiogenic treatment response in advanced colorectal cancer as well as in metastatic renal-cell carcinoma. ${ }^{20} 35$ rs9513070 and three other SNPs in linkage disequilibrium have been linked to a decreased VEGF pathway activity. $^{20}$

Finally, three SNPs near VEGFC were significantly associated with response to anti-VEGF treatment after 12 months (table 2). Of these, rs12054587 is of note, as the alternative $\mathrm{A}$ allele at this locus is associated with a lower VEGFC expression in a recent data release of the Genotype-Tissue Expression project. ${ }^{36}$

Four major limitations of our study need to be highlighted. First one is its retrospective nature: the treatment protocol may have varied between the patients treated in different years, leading to heterogeneity in treatment responses between included patients. Nevertheless, there was no difference in response rate between the different years in which patients started treatment (not shown). Heterogeneity in the drug applied may affected associations at 12 months, explaining the limited overlap. At 3 months however, all patients received a similar regimen and differences in the type of drug have not confounded the analyses.

Second, we note that CFT, morphological changes and visual acuity were invariably documented during monthly visits to the clinic, but before patients receive their monthly injection. This implies that non-responding patients may have shown response in the days and weeks following their injection at month 2 , but that this response had disappeared at the time of their next attendance.

Third important limitation is that $p$ values did not survive correction for multiple testing. As mentioned, our results are supported by previous studies, which show similar SNPs affecting response to anti-VEGF therapies, as well as by a reanalysis of a previously published Japanese cohort which showed a similar trend but where again no $\mathrm{p}$ values survived multiple testing correction. Hence, our data should be interpreted with caution given their susceptibility to higher false discovery rates. Indeed, the overlap of the seven associations detected after 3 months with associations after 12 months was limited: Only one SNP, rs7691507, was also significant $(p<0.05)$ after 12 months, while two other SNPs were borderline significant (rs3025035 and rs2305945, with P-values of respectively, 0.057 and 0.053 ) and four SNPs were not significant. Also, the overlap with the structural changes at 3 months was not tremendous: $\mathrm{p}<0.05$ for rs55667289 and rs9513070, and borderline significance for rs 7691507 and rs5758223, $\mathrm{p}=0.050$ and $\mathrm{p}=0.054$.

A final limitation is the poor replication. Indeed, the Japanese cohort showed a similar trend, but no $\mathrm{p}$ values survived multiple testing correction. This may be due in part to differences in the genetic make-up of this population, being different from the discovery cohort of European ancestry, as well as due to the fact that response in the validation cohort was assess by changes in visual acuity, while responses in the discovery cohort data were defined by CFT changes.

In conclusion, our study provides further insight into the pharmacogenetics of treatment response to anti-VEGF therapies, by identifying novel SNPs that are putatively associated with lack of treatment response. These variants seem to reduce the overall activity of the VEGF pathway. This suggests that neovascularisation in these non-responsive patients could be driven by angiogenic pathways other than the VEGF pathway. For example, dysregulation of micro RNA signalling and PGF-specific alterations have been reported in retinal neoangiogenesis. ${ }^{37} 38$ Hence, by defining mechanisms leading to resistance to the current anti-VEGF agents, this study may aid in the development of new therapies for these non-responding patients, as well as in the stratification of ARMD patient populations for their predicted response. Accumulating data from the genetic analysis of different populations with ARMD will further advance our understanding of the biological pathways that underlie treatment resistance and may enable the development of novel therapies and novel regimes for existing therapies. Moreover, new molecules targeting VEGFC and VEGFD are currently being evaluated for treatment efficacy, thus underlining the possible role of the VEGFC and VEGFD pathways we identified here in neovascular ARMD. 
Author affiliations

'Department of Ophthalmology, Ghent University Hospital, Ghent University, Ghent, Belgium

${ }^{2}$ Ophthalmology, University Hospital Brugmann, Université Libre de Bruxelles,

Brussels, Belgium

${ }^{3}$ Department of Ophthalmology and Visual Sciences, Kyoto University Graduate

School of Medicine, Kyoto, Japan

${ }^{4}$ Department of Ophthalmology, Otsu Red Cross Hospital, Otsu, Japan

5 Laboratory of Translational Genetics, Department of Human Genetics, KU Leuven, Leuven, Belgium

${ }^{6}$ Laboratory of Functional Epigenetics, Department of Human Genetics, KU Leuven, Leuven, Belgium

${ }^{7}$ VIB Center for Cancer Biology, Leuven, Belgium

Acknowledgements The authors would like to thank Thomas Van Brussel for his technical assistance, patient advisers for their contribution and the reviewers for providing essential critical comments.

Collaborators Kenji Yamashiro; Keisuke Mori; Shigeru Honda; Mariko Kano; Yasuo Yanagi; Akira Obana; Yoichi Sakurada; Taku Sato; Yoshimi Nagai; Taiichi Hikichi; Yasushi Kataoka; Chikako Hara; Yasurou Koyama; Hideki Koizumi; Munemitsu Yoshikawa; Masahiro Miyake; Isao Nakata; Takashi Tsuchihashi; Kuniko HorieInoue; Wataru Matsumiya; Masashi Ogasawara; Ryo Obata; Seigo Yoneyama; Hidetaka Matsumoto; Masayuki Ohnaka; Hirokuni Kitamei; Kaori Sayanagi; Sotaro Ooto; Hiroshi Tamura; Akio Oishi; Sho Kabasawa; Kazuhiro Ueyama; Akiko Miki; Naoshi Kondo; Hiroaki Bessho; Masaaki, Saito; Hidenori Takahashi; Xue Tan; Keiko Azuma; Wataru Kikushima; Ryo Mukai; Akihiro Ohira; Fumi Gomi; Kazunori Miyata; Kanji Takahashi; Shoji Kishi; Hiroyuki lijima; Tetsuju Sekiryu; Tomohiro lida; Takuya Awata; Satoshi Inoue; Ryo Yamada; Fumihiko Matsuda; Akitaka Tsujikawa; Akira Negi; Shin Yoneya; Takeshi Iwata; Nagahisa Yoshimura.

Contributors IB: design of the study, conducted the study, writing manuscript, submission, overall content of the study; LP: clinical work, design of the study, writing of the manuscript; JC and PC: clinical work; AE: collected data; YH, MM, $\mathrm{KY}$ and Angel group: Japanese cohort; BT: conducted the study, analysis, writing manuscript, DL: design of the study, funding, writing manuscript.

Funding The study was funded by Brugmann Foundation grant.

Competing interests None declared.

Patient consent for publication Obtained.

Ethics approval This study was approved by the local ethics Committee (Le Comité d'Ethique du CHU Brugmann), under approval number CE2013/53.

Provenance and peer review Not commissioned; externally peer reviewed.

Data availability statement Data are available upon request

Open access This is an open access article distributed in accordance with the Creative Commons Attribution Non Commercial (CC BY-NC 4.0) license, which permits others to distribute, remix, adapt, build upon this work non-commercially, and license their derivative works on different terms, provided the original work is properly cited, appropriate credit is given, any changes made indicated, and the use is non-commercial. See: http://creativecommons.org/licenses/by-nc/4.0/.

ORCID iD

Irina Balikova http://orcid.org/0000-0002-7487-2288

\section{REFERENCES}

1 Friedman DS, O'Colmain BJ, Muñoz B, et al. Prevalence of agerelated macular degeneration in the United States. Arch Ophthalmol 2004;122:564-72.

2 Chakravarthy U, Evans J, Rosenfeld PJ. Age related macular degeneration. BMJ 2010;340:c981.

3 Wang $\mathrm{H}$, Hartnett ME. Regulation of signaling events involved in the pathophysiology of neovascular AMD. Mol Vis 2016;22:189-202.

4 Rosenfeld PJ, Brown DM, Heier JS, et al. Ranibizumab for neovascular age-related macular degeneration. N Engl J Med 2006;355:1419-31.

5 Chakravarthy U, Harding SP, Rogers CA, et al. A randomised controlled trial to assess the clinical effectiveness and costeffectiveness of alternative treatments to inhibit VEGF in agerelated choroidal neovascularisation (IVAN). Health Technol Assess 2015;19:1-298.
6 Heier JS, Brown DM, Chong V, et al. Intravitreal aflibercept (VEGF trap-eye) in wet age-related macular degeneration. Ophthalmology 2012;119:2537-48

7 Brown DM, Michels M, Kaiser PK, et al. Ranibizumab versus verteporfin photodynamic therapy for neovascular age-related macular degeneration: two-year results of the anchor study. Ophthalmology 2009;116:57-65.

8 Lorés-Motta L, de Jong EK, den Hollander Al. Exploring the use of molecular biomarkers for precision medicine in age-related macular degeneration. Mol Diagn Ther 2018;22:315-43.

9 Cobos E, Recalde S, Anter J, et al. Association between CFH, CFB, ARMS2, SERPINF1, VEGFR1 and VEGF polymorphisms and anatomical and functional response to ranibizumab treatment in neovascular age-related macular degeneration. Acta Ophthalmol 2018;96:e201-12.

10 Abedi F, Wickremasinghe S, Richardson AJ, et al. Genetic influences on the outcome of anti-vascular endothelial growth factor treatment in neovascular age-related macular degeneration. Ophthalmology 2013;120:1641-8.

11 Hong N, Shen Y, Yu C-Y, et al. Association of the polymorphism $\mathrm{Y} 402 \mathrm{H}$ in the CFH gene with response to anti-VEGF treatment in age-related macular degeneration: a systematic review and metaanalysis. Acta Ophthalmol 2016;94:334-45.

12 Hagstrom SA, Ying G-S, Pauer GJT, et al. Pharmacogenetics for genes associated with age-related macular degeneration in the comparison of AMD treatments trials (CATT). Ophthalmology 2013;120:593-9.

13 Hagstrom SA, Ying G-shuang, Maguire MG, et al. Vegfr2 gene polymorphisms and response to anti-vascular endothelial growth factor therapy in age-related macular degeneration. Ophthalmology 2015;122:1563-8.

14 Hermann MM, van Asten F, Muether PS, et al. Polymorphisms in vascular endothelial growth factor receptor 2 are associated with better response rates to ranibizumab treatment in age-related macular degeneration. Ophthalmology 2014;121:905-10.

15 Finger RP, Wickremasinghe SS, Baird PN, et al. Predictors of anti-VEGF treatment response in neovascular age-related macular degeneration. Surv Ophthalmol 2014;59:1-18.

$16 \mathrm{Wu} \mathrm{M}$, Xiong $\mathrm{H}, \mathrm{Xu}$ Y, et al. Association between VEGF-A and VEGFR-2 polymorphisms and response to treatment of neovascular AMD with anti-VEGF agents: a meta-analysis. $\mathrm{Br} J$ Ophthalmol 2017;101:976-84.

17 Riaz M, Lorés-Motta L, Richardson AJ, et al. Gwas study using DNA pooling strategy identifies association of variant rs 4910623 in OR52B4 gene with anti-VEGF treatment response in age-related macular degeneration. Sci Rep 2016;6:37924.

18 Akiyama M, Takahashi A, Momozawa Y, et al. Genome-ide association study suggests four variants influencing outcomes with ranibizumab therapy in exudative age-related macular degeneration. $J$ Hum Genet 2018;63:1083-91.

19 Jubb AM, Harris AL. Biomarkers to predict the clinical efficacy of bevacizumab in cancer. Lancet Oncol 2010;11:1172-83.

20 Lambrechts D, Claes B, Delmar P, et al. VEGF pathway genetic variants as biomarkers of treatment outcome with bevacizumab: an analysis of data from the AViTA and AVOREN randomised trials. Lancet Oncol 2012;13:724-33.

21 Mitchell P, Korobelnik J-F, Lanzetta P, et al. Ranibizumab (Lucentis) in neovascular age-related macular degeneration: evidence from clinical trials. Br J Ophthalmol 2010;94:2-13.

22 Lalwani GA, Rosenfeld PJ, Fung AE, et al. A variable-dosing regimen with intravitreal ranibizumab for neovascular age-related macular degeneration: year 2 of the PrONTO study. Am J Ophthalmol 2009;148:43-58. e41.

23 Yamashiro K, Mori K, Honda S, et al. A prospective multicenter study on genome wide associations to ranibizumab treatment outcome for age-related macular degeneration. Sci Rep 2017;7:9196.

24 Ibrahim MA, Sepah YJ, Symons RCA, et al. Spectral- and timedomain optical coherence tomography measurements of macular thickness in normal eyes and in eyes with diabetic macular edema. Eye 2012;26:454-62.

25 Yang WR, Ardeljan D, Pacyna CN, et al. Squire reveals locus-specific regulation of interspersed repeat expression. Nucleic Acids Res 2019;47:e27

26 Simader C, Ritter M, Bolz M, et al. Morphologic parameters relevant for visual outcome during anti-angiogenic therapy of neovascular age-related macular degeneration. Ophthalmology 2014:121:1237-45.

27 Jaffe GJ, Martin DF, Toth CA, et al. Macular morphology and visual acuity in the comparison of age-related macular degeneration treatments trials. Ophthalmology 2013;120:1860-70. 
28 Garcia-Donas J, Leandro-García LJ, González Del Alba A, et al. Prospective study assessing hypoxia-related proteins as markers for the outcome of treatment with sunitinib in advanced clear-cell renal cell carcinoma. Ann Oncol 2013;24:2409-14.

29 Deng Y, Zhang X, Simons M. Molecular controls of lymphatic VEGFR3 signaling. Arterioscler Thromb Vasc Biol 2015;35:421-9.

30 Karkkainen MJ, Ferrell RE, Lawrence EC, et al. Missense mutations interfere with VEGFR-3 signalling in primary lymphoedema. Nat Genet 2000;25:153-9.

31 Suzuki H, Watabe T, Kato M, et al. Roles of vascular endothelial growth factor receptor 3 signaling in differentiation of mouse embryonic stem cell-derived vascular progenitor cells into endothelial cells. Blood 2005;105:2372-9.

32 Heinolainen K, Karaman S, D'Amico G, et al. VEGFR3 modulates vascular permeability by controlling VEGF/VEGFR2 signaling. Circ Res 2017;120:1414-25.
33 Fang AM, Lee AY, Kulkarni M, et al. Polymorphisms in the VEGFA and VEGFR-2 genes and neovascular age-related macular degeneration. Mol Vis 2009;15:2710.

34 Pabst S, Karpushova A, Diaz-Lacava A, et al. VEGF gene haplotypes are associated with sarcoidosis. Chest 2010;137:156-63.

35 Sohn BS, Park SJ, Kim JE, et al. Single-nucleotide polymorphisms in the vascular endothelial growth factor pathway and outcomes of patients treated with first-line cytotoxic chemotherapy combined with bevacizumab for advanced colorectal cancer. Oncology 2014;87:280-92.

36 Battle A, Brown CD, Engelhardt BE, et al. Genetic effects on gene expression across human tissues. Nature 2017;550:204-13.

37 Askou AL, Alsing S, Holmgaard A, et al. Dissecting microRNA dysregulation in age-related macular degeneration: new targets for eye gene therapy. Acta Ophthalmol 2018;96:9-23.

38 Nguyen QD, De Falco S, Behar-Cohen F, et al. Placental growth factor and its potential role in diabetic retinopathy and other ocular neovascular diseases. Acta Ophthalmol 2018;96:e1-9. 